\title{
Exponential Stability Region Estimates for the State-Dependent Riccati Equation Controllers
}

\author{
Insu Chang and Soon-Jo Chung
}

\begin{abstract}
We investigate the nonlinear exponential stability of the State-Dependent Riccati Equation (SDRE)-based control. The SDRE technique is a nonlinear control method, which has emerged since the mid 1990's and has been applied to a wide range of nonlinear control problems. Despite the systematic method of SDRE, it is difficult to prove stability because the general analytic solution to the SDRE is not known. Some notable prior work has shown local asymptotic stability of SDRE by using numerical and analytical methods. In this paper, we introduce a new strategy, based on contraction analysis, to estimate the exponential stability region for SDRE controlled systems. Examples demonstrate the superiority of the proposed method.
\end{abstract}

\section{INTRODUCTION}

The State-Dependent Riccati Equation (SDRE) techniques are general design methods that provide a systematic and effective means of designing nonlinear controllers, observers, and filters [9]. SDRE control overcomes many of the difficulties and weaknesses of the existing methodologies such as feedback linearization, and delivers computationally efficient algorithms that are highly effective in a variety of practical applications [8] such as systems with hard bounds on control and unstable non-minimum phase systems [21].

SDRE approach to nonlinear systems can be established by using state-dependent coefficient matrices that represent a nonlinear system's dynamics in a manner which resembles linear dynamics. Then, the state-dependent Riccati equations are generated for a feedback law for the nonlinear system. It is systematic and easy to implement, so that it has been applied to various nonlinear control problems: autopilot design [11], guidance law design [10], satellite attitude and orbit control [5], [6], [15], missile guidance and control systems [20], an underactuated robot [12], a magnetically levitated ball [13], helicopters [2], a pendulum problem [26], etc.

Although the SDRE technique has been evaluated successfully, the computation of the stability region for the SDRE-controlled systems is an open problem. An analytic solution to the SDRE is generally not known [4]. This is because the algebraic state-dependent Riccati equation is solved numerically. There have been many attempts to solve this problem. Some notable results are as follows: Erdem and Alleyne proposed an analytic solution to estimate the asymptotic stability region for the SDRE controllers [13]. In order to use this method, however, the SDRE controlled

The authors are with the Department of Aerospace Engineering, University of Illinois at Urbana-Champaign, Urbana, IL 61801, USA. Email: chang162@illinois.edu, sjchung@illinois.edu. system should have a dynamic model matrix with less than 3 dimensions. Erdem and Alleyne suggested another method to satisfy higher-order dynamic systems by using vector norms [14]. By determining the overvaluing matrix for the given dynamic system, the method shows the maximum boundary for the SDRE-controlled dynamic systems. However, this method is complicated from the computational standpoint for medium and high order systems [4]. Langson and Alleyne tried to find a stable upper boundary for the SDRE controlled system [17]. Seiler [23] introduced a method to estimate the stability region by turning the stability problem into a semi-definite programming problem, called the Sum-ofSquares (SOS) program [22], [1]. By using the SOS program, largest values of decision variables satisfying the Lyapunov stability condition are found. However, if the dimension of the system matrix is greater than two, it takes a large set of internal variables to find the optimal values for maximizing the stability region for the SDRE feedback system. Another numerical method was suggested by Bracci et al. [4]. This procedure is an alternative to the method proposed by Erdem and Alleyne [14], and is based on the Lyapunov local stability theorem [25].

One notable research [19] proposed a stability test for determining the size of the region on which large-scale asymptotic stability holds for the SDRE algorithm by using the geometrical construction of a viscosity-type Lyapunov function. The stability region estimates for the SDRE feedback are very close to the true domain of attraction than conservative estimates in the existing literature. However, all the aforementioned methods show only the asymptotic stability of the nonlinear systems controlled by the SDRE controllers. The objective of this work is to suggest a numerical method to find the exponential stability region along with the corresponding convergence rate. It should be noted that exponential stability is stronger than asymptotic stability and offers better performance and robustness in the presence of perturbations [7].

In this paper, we introduce a new strategy to estimate the exponential stability region for the SDRE feedback systems by using contraction theory [18]. The contraction theory is a relatively new and very powerful tool for proving exponential stability of the nonlinear systems. By applying contraction analysis to the SDRE controlled systems, we can guarantee the exponential stability of the system, while most of the aforementioned results in the literature guarantees only asymptotic stability. Some examples show the superiority of the proposed method, in that we can check the exponential stability region, which is usually more accurate 
than that demonstrated by the existing literature, and also how accurately the system's errors converge to zero with the information of convergence rate.

The organization of this paper is as follows: In Section II, preliminaries of the SDRE control are discussed. A brief introduction to contraction analysis is presented in Section III. The stability proof of the SDRE controlled systems is described in Section IV. In Section V, two numerical examples are presented to compare the results with other numerical methods. Finally, in Section VI, concluding remarks are stated.

\section{OVERVIEW OF THE SDRE}

Consider the deterministic, infinite-horizon nonlinear optimal regulation problem, where the system is full-state observable, autonomous, nonlinear in the state, and affine in the input, represented in the form [8]

$$
\dot{x}(t)=f(x)+B(x) u(t), \quad x(0)=x_{0}
$$

where $x \in \mathbb{R}^{n}$ is the state vector, $u \in \mathbb{R}^{m}$ is the input vector.

The SDRE technique is a nonlinear control design method for the direct construction of nonlinear feedback controllers. Through the state-dependent coefficient (SDC) factorization, system designers can represent the nonlinear equations of motion as linear structures with state-dependent coefficients. Then, the LQR technique can be applied to this statedependent state-space equation. Thus, the following procedure is similar to the LQR method, except that all matrices may depend on the states. Based on this concept, the statespace equation for the nonlinear system described in (1) can be expressed as a linear-like state-space equation using direct SDC factorization as:

$$
\dot{x}=A(x) x+B(x) u
$$

where the factorization for $f(x)=A(x) x$ is possible if and only if $f(0)=0$ and $f(x)$ is continuously differentiable. Note that $A(x)$ is not a unique matrix because there could be many possible choices in the direct SDC factorization [9]. For this system, the SDRE technique finds an input $u(t)$ that approximately minimizes the following performance index:

$$
J=\frac{1}{2} \int_{0}^{\infty}\left(x^{T} Q(x) x+u^{T} R(x) u\right) \mathrm{d} t
$$

where $Q(x)$ is a positive semi-definite matrix with quadratic form and $R(x)$ is a positive definite matrix with quadratic form for all $x$. Also, it is assumed that $f(0)=0$ and $B(x) \neq 0$. It should be noted that $Q(x)$ and $R(x)$ are not only allowed to be constant, but can also be varied as functions of states. As these state-dependent matrices are applied to the algebraic Riccati equation (ARE), the following statedependent Riccati equation is obtained [9]:

$$
\begin{aligned}
P(x) A(x) & +A^{T}(x) P(x)+Q(x) \\
& -P(x) B(x) R^{-1}(x) B^{T}(x) P(x)=0
\end{aligned}
$$

The optimal feedback control gain matrix, which is a state-dependent $m \times n$ variable gain matrix, and the $m \times 1$ input control can be calculated in the same way as the LQR technique except for the state dependence:

$$
\begin{aligned}
K(x) & =R^{-1}(x) B^{T}(x) P(x) \\
u & =-K(x) x
\end{aligned}
$$

where $P(x) \in \mathbb{R}^{n \times n}$ is the unique positive-definite solution of the SDRE (4).

As with the LQR technique, the SDRE technique also constructs a closed-loop system with direct state feedback controller $u(t)$ as a regulator. However, the feedback gain, $K(x)$, of the SDRE technique depends on the states. Hence, state-dependent control inputs are applied to the plant. Because the state-space equation (2) should be computed for every state and control input, (4) and (5) should be calculated at each time step. Because the SDRE technique can be considered as the LQR method for each time step, the matrix $P(x)$ in (4) becomes a unique solution of the algebraic Riccati equation at the particular state, $x(t)$, which means it has constant values at each given state. Therefore, solving the ARE in (4) for each $x$ is feasible and can be done either on-line or off-line [12].

Controllability is critical because it is a sufficient condition for the existence of a solution to the SDRE. In general, a linear time-invariant system is controllable if and only if the $n \times n m$ controllability matrix $W_{\text {ctrl }}$ has full rank (that is, $\left.\operatorname{rank}\left(W_{\text {ctrl }}\right)=n\right)$. The controllability of the SDRE can be determined by pointwise controllability $\left(W_{\text {ctrl }}(x)\right)$ of the SDC factorization

$$
W_{\text {ctrl }}(x)=\left[\begin{array}{lllll}
B(x) & A(x) B(x) & A^{2}(x) B(x) & \cdots & A^{n-1}(x) B(x)
\end{array}\right] .
$$

Thus, the selection of $(A(x)$ and $B(x))$ can affect the controllability of the system.

\section{Contraction Theory}

The new method proposed in this paper is motivated by contraction analysis, a relatively new nonlinear stability tool for exponential stability for the nonlinear systems. It is a generalized version of Krasovskii's theorem [25], which provides a sufficient, asymptotic convergence result. Readers are referred to [18] for more detailed information about contraction analysis.

Consider general deterministic systems of the form

$$
\dot{x}(t)=f(x, u(x, t), t)
$$

where $f: \mathbb{R}^{n} \times \mathbb{R}^{m} \times \mathbb{R} \longmapsto \mathbb{R}^{n}$ is a nonlinear vector function and $x \in \mathbb{R}^{n}$ is the state vector. This nonlinear system can be thought of as an $n$-dimensional fluid flow, where $\dot{x}$ is the $n$ dimensional "velocity" vector at the $n$-dimensional position $x$ and time $t$. Assuming that $f(x, u(x, t), t)$ is continuously differentiable, the exact differential relation can be obtained by (7):

$$
\delta \dot{x}(t)=\frac{\partial f}{\partial x}(x, u(x, t), t) \delta x
$$

where $\delta x$ is a virtual displacement of the systems. Note that $\delta x$ defines a linear tangent differential form, and $\delta x^{T} \delta x$ 
the associated quadratic tangent form, both of which are differentiable with respect to time $t$.

Consider two neighboring trajectories in the flow field (7), and the virtual displacement $\delta x$ between them. The squared distance (quadratic virtual length) between these two trajectories can be defined as $\delta x^{T} \delta x$, leading from (8) to the rate of change

$$
\frac{\mathrm{d}}{\mathrm{d} t}\left(\delta x^{T} \delta x\right)=2 \delta x^{T} \delta \dot{x}=2 \delta x^{T} \frac{\partial f}{\partial x} \delta x .
$$

Denoting by $\lambda_{\max }(x, t)$ the largest eigenvalue of the symmetric part of the Jacobian $\partial f / \partial x$, we have

$$
\frac{\mathrm{d}}{\mathrm{d} t}\left(\delta x^{T} \delta x\right) \leq 2 \lambda_{\max } \delta x^{T} \delta x
$$

and hence,

$$
\|\delta x\| \leq\left\|\delta x_{0}\right\| e^{\int_{0}^{t} \lambda_{\max }(x, t) \mathrm{d} t}
$$

Assuming that $\lambda_{\max }$ is uniformly strictly negative, then from (11) any infinitesimal length $\|\delta x\|$ converges exponentially to zero.

\section{Generalized Contraction Analysis}

The line vector $\delta x$ defined in (8) can also be expressed using the differential coordinate transformation [18], and leads to a generalization of the previous definition of squared length as

$$
\delta z=\Theta(x, t) \delta x, \quad \delta z^{T} \delta z=\delta x^{T} M \delta x
$$

where $\Theta(x, t)$ and $M=\Theta^{T} \Theta$ denote a square matrix and a symmetric and continuously differentiable metric, respectively. Therefore, exponential convergence of $\delta z$ to 0 implies exponential convergence of $\delta x$ to 0 .

The time derivative of $\delta z=\Theta \delta x$ can be computed as

$$
\begin{aligned}
\frac{\mathrm{d}}{\mathrm{d} t} \delta z & =\dot{\Theta} \delta x+\Theta \delta \dot{x} \\
& =(\dot{\Theta}+\Theta \partial f / \partial x) \Theta^{-1} \delta z \triangleq H \delta z
\end{aligned}
$$

The rate of change of squared length can be written

$$
\frac{\mathrm{d}}{\mathrm{d} t}\left(\delta z^{T} \delta z\right)=2 \delta z^{T} H \delta z
$$

Therefore, if there exists a $\gamma>0$, such that the symmetric part of $H$ is negative definite, that is,

$$
\left(H+H^{T}\right) / 2<-\gamma I,
$$

then the system is exponentially stable. It is helpful to recall that $H=H(x, t)$.

By using the characteristics of contraction analysis, we will estimate the exponential stability region for the SDRE controlled systems in the next section.

\section{Stability Region Estimate for the SDRE}

By using the concepts of the aforementioned contraction analysis, the new method will be introduced in this section. Prior to that, we need to derive the necessary mathematical model.

Given the nonlinear equation (7) under the assumption of an autonomous nonlinear equation, the equation can be rewritten in the form (2) by applying the SDC factorization. Moreover, by applying the control law (5) to the SDC factorization, the closed-loop form can be obtained as

$$
\begin{aligned}
\dot{x} & =(A(x)-B(x) K(x)) x \\
& =\left(A(x)-B(x) R^{-1}(x) B^{T}(x) P(x)\right) x \\
& \triangleq A_{\mathrm{cl}}(x) x .
\end{aligned}
$$

Note that some prior work states that Hurwitz $A_{\mathrm{cl}}(x)$ often times is good enough for the closed-loop stability of the system [24]. In general, however, we cannot assure stability of the closed-loop system by checking the pointwise Hurwiz condition of $A_{\mathrm{cl}}(x)$. This is one of the motivations of the current study.

For the virtual length analysis for the contraction analysis, the quadratic virtual length can be defined as $\delta x^{T} M(x) \delta x$ where $M(x)$ is a symmetric positive metric, defined in (12). The rate of change of the quadratic virtual length is

$$
\begin{aligned}
\frac{\mathrm{d}}{\mathrm{d} t}\left(\delta x^{T} M(x) \delta x\right) & =\delta \dot{x}^{T} M(x) \delta x+\delta x^{T} \dot{M}(x) \delta x+\delta x^{T} M(x) \delta \dot{x} \\
& =\delta x^{T}\left(F^{T}(x) M(x)+M(x) F(x)+\dot{M}(x)\right) \delta x
\end{aligned}
$$

where $F(x)$ is defined as $F(x)=A_{\mathrm{cl}}(x)+\frac{\partial A_{\mathrm{cl}}(x)}{\partial x} x$.

Therefore, given $x \in \mathbb{R}^{n}$ and given the local region $B_{R}(x)$ by the states, if there exists $\gamma(x)>0$, such that

$$
F^{T}(x) M(x)+M(x) F(x)+\dot{M}(x)<-\gamma(x) M(x),
$$

then the quadratic virtual length satisfies the following condition

$$
\frac{\mathrm{d}}{\mathrm{d} t}\left(\delta x^{T} M(x) \delta x\right)<-\gamma(x) \delta x^{T} M(x) \delta x,
$$

which implies that the system is exponentially stable on $B_{R}(x)$ by the condition (11). It should be noted $\dot{M}(x)$ is assumed to be sufficiently small with sufficiently small $\dot{x}$ and bounded $\partial M(x) / \partial x$. Therefore, in the SDRE controlled system, (18) can be relaxed to

$$
F^{T}(x) M(x)+M(x) F(x)<-\gamma(x) M(x) \leq-\beta I
$$

for some $\beta>0$.

Therefore, the exponential stability region can be estimated by solving the inequality (20) and obtaining $\gamma(x)$ in the local region for each state. This equation is similar to the Lyapunov inequality [3] except the fact that (20) is solved in each state. In order to solve (20), we can use two constraints: $\gamma(x)>0$ and the symmetric positive definiteness of $M(x)$, i.e., the leading principal minors of $M(x)$ must be positive [3]. Unlike the aforementioned prior work, the proposed method can show how fast the SDRE controllers stabilize 
the nonlinear system exponentially - $\gamma(x)$ is the local exponential convergence rate.

In the next section, two examples are described to compare the proposed method with a previous method by Bracci et al..

\section{EXAMPLES}

In this section, two different examples are presented: the first one discusses an analytic nonlinear open-loop system [4] and the other presents a nonlinear feedback control system [24] with the SDRE controller. In the first example, which gives the analytic results, we will evaluate the superiority and the effectiveness of the proposed method.

\section{A. First Example}

First, consider the following nonlinear autonomous system:

$$
\dot{x}=A(x) x=\left[\begin{array}{cc}
-3+x_{1}^{2} & x_{2} \sin \left(x_{2}\right) \\
4 x_{1}^{2} \cos \left(x_{2}\right) & -3+x_{2}^{2}
\end{array}\right] x .
$$

The asymptotic stability region can be found by the method of Bracci et al. [4]. Briefly the procedure for this method is as follows: The Lyapunov function can be chosen to be

$$
V(x)=\frac{1}{2} x^{T} x=\frac{1}{2}\left(x_{1}^{2}+x_{2}^{2}\right) .
$$

The time derivative of the Lyapunov function (22) is

$$
\dot{V}(x)=x^{T} \dot{x}=x^{T} A(x) x .
$$

Therefore, the asymptotic stability region can be found by checking the Lyapunov local stability theorem [25]: the region satisfying $V>0$ and $\dot{V}<0$.

Now, let us apply the proposed method to estimate the stability region. From (17), the partial derivative of $A(x) x$ for the given system is needed:

$$
\begin{aligned}
\frac{\partial(A(x) x)}{\partial x} & =A(x)+\frac{\partial(A(x))}{\partial x} x \\
& =\left[\begin{array}{cc}
-3+3 x_{1}^{2} & 2 x_{2} \sin \left(x_{2}\right)+x_{2}^{2} \cos \left(x_{2}\right) \\
12 x_{1}^{2} \cos \left(x_{2}\right) & -3+3 x_{2}^{2}-4 x_{1}^{3} \sin \left(x_{2}\right)
\end{array}\right] .
\end{aligned}
$$

In order to find a reasonable symmetric positive metric, $M(x)$, for stability region estimates by the proposed method, let us consider the condition (20),

$$
F^{T}(x) M(x)+M(x) F(x)<-\gamma(x) M(x),
$$

where $F(x)=A(x)+\frac{\partial(A(x))}{\partial x} x$, as in (17) and (24). Therefore, if there exists $\gamma(x)>0$ for the given states, then one can say that the local region is locally exponentially stable. The union of the local stability regions approximates the estimated stability region for the system.

Fig. 1 shows the stability region for the nonlinear system (21). Fig. 1(a) illustrates the stability region estimates by the proposed method (the black circle) and the method by Bracci et al. [4] (the gray circle). The figure shows that the stability region estimated by the proposed method is larger than that by Bracci et al.. This is mainly because we use a statedependent $M(x)$, while Bracci et al. use a constant $M$, which was obtained at the equilibrium point, for constructing the

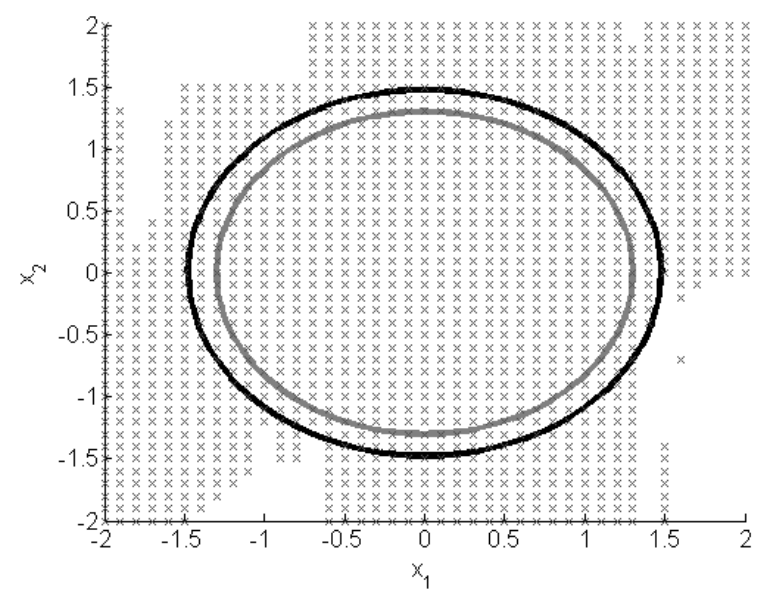

(a) Stability region estimated by the proposed method (The black circle describes the stability region by the proposed method and the gray circle denotes the result by Bracci et al.)

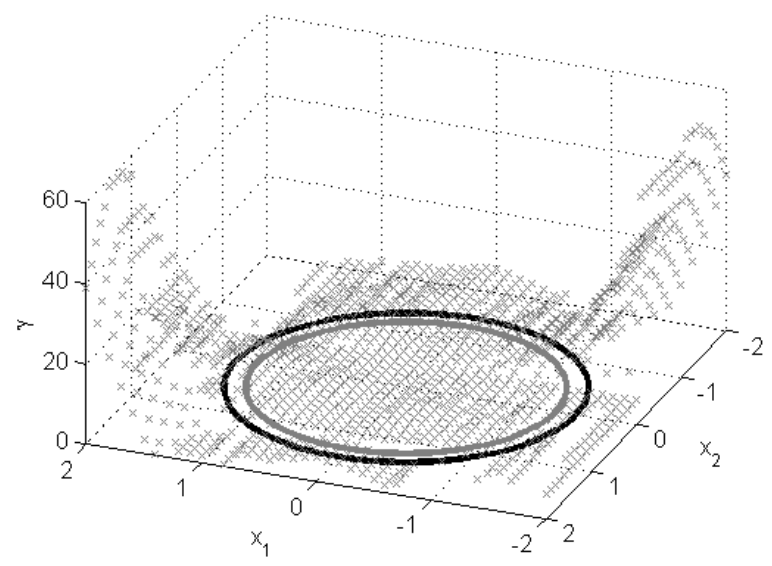

(b) Estimated values of the convergence rate, $\gamma$, for the proposed method

Fig. 1. Comparison of the stability region estimates for Example 1

Lyapunov function, which may fail to show more accurate results. Fig. 1(b) shows the additional result for the proposed method. The convergence rate $(\gamma)$ inside the stability region estimate has relatively similar but lower values than those outside the region.

\section{B. Second Example}

The second example is for a simple nonlinear feedback control from [24]. Consider the second-order nonlinear system:

$$
\dot{x}=A(x) x+B u=\left[\begin{array}{cc}
x_{1} & 1 \\
0 & 0
\end{array}\right] x+\left[\begin{array}{l}
0 \\
1
\end{array}\right] u .
$$

For simplicity, let us assume that the weighting matrices $Q(x)$ and $R(x)$, which are used in the algebraic Riccati equation as well as in the Lypunov equation [16] for the method by Bracci et al., are constants such that

$$
Q=\operatorname{diag}(100,100), \quad R=1 .
$$

In order to apply the method by Bracci et al., we need to solve the Lyapunov equation, which is used to construct 


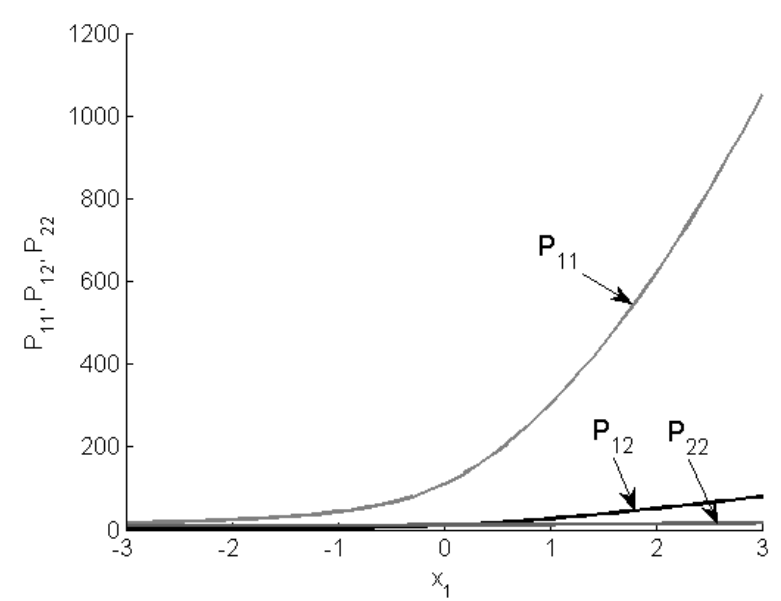

Fig. 2. Solutions of the algebraic Riccati equation $(P(x))$ with respect to $x_{1}$ in the Example 2. The solutions do not depend on $x_{2}$.

the Lyapunov function candidate. Readers are referred to [4] for the detailed procedure. The solution of the Lyapunov equation in the equilibrium point can be calculated as:

$$
A_{\mathrm{cl}, 0}^{T} M^{\prime}+M^{\prime} A_{\mathrm{cl}, 0}=-Q
$$

where $A_{\mathrm{cl}, 0}$ denote the Jacobian of the nonlinear system (26) in the equilibrium point.

Now, let us apply the proposed method to this nonlinear system to estimate the exponential stability region. For this purpose, we need to derive the analytic equation for the partial derivative in (17). i.e.,

$$
\begin{aligned}
F(x) & =A_{\mathrm{cl}}(x)+\frac{\partial A_{\mathrm{cl}}(x)}{\partial x} x \\
& =\left[\begin{array}{cc}
2 x_{1} & 1 \\
-p_{12} & -p_{22}
\end{array}\right]-\left[\begin{array}{cc}
0 & 0 \\
\frac{\partial p_{12}}{\partial x_{1}} x_{1}+\frac{\partial p_{22}}{\partial x_{1}} x_{2} & \frac{\partial p_{12}}{\partial x_{2}} x_{1}+\frac{\partial p_{22}}{\partial x_{2}} x_{2}
\end{array}\right]
\end{aligned}
$$

where $p_{12}$ and $p_{22}$ denote the elements of $P(x)$, which is the unique solution of the algebraic state-dependent Riccati equation (4), and which can be obtained by solving the algebraic Riccati equation at the given state values, since a closed-form solution is generally not available. The terms $\frac{\partial p_{12}}{\partial x_{1}}, \frac{\partial p_{22}}{\partial x_{1}}, \frac{\partial p_{12}}{\partial x_{2}}$, and $\frac{\partial p_{22}}{\partial x_{2}}$ can be obtained from the numerical results in Fig. 2, which shows the solutions of the elements of $P(x)$ with respect to the state $x_{1}$. The solutions $p_{11}, p_{12}$, and $p_{22}$ can be obtained by solving the ARE, or element-wise,

$$
\begin{aligned}
& 100-p_{12}(x)^{2}+2 p_{11}(x) x_{1}=0 \\
& p_{11}(x)-p_{12}(x) p_{22}(x)+p_{12}(x) x_{1}=0 \\
& 2 p_{12}(x)-p_{22}(x)^{2}+100=0 .
\end{aligned}
$$

It should be noted that the proposed method considers the last term in (29) and state-dependent $M(x)$, while the aforementioned prior work has ignored these two terms. Moreover, finding $P(x)$ numerically is sufficient to find the $\partial P / \partial x$ for a higher-order system.

Fig. 3 shows the results for the estimated stability regions. Fig. 3(a) describes the stability region estimate for the method by Bracci et al.. The gridded region and the

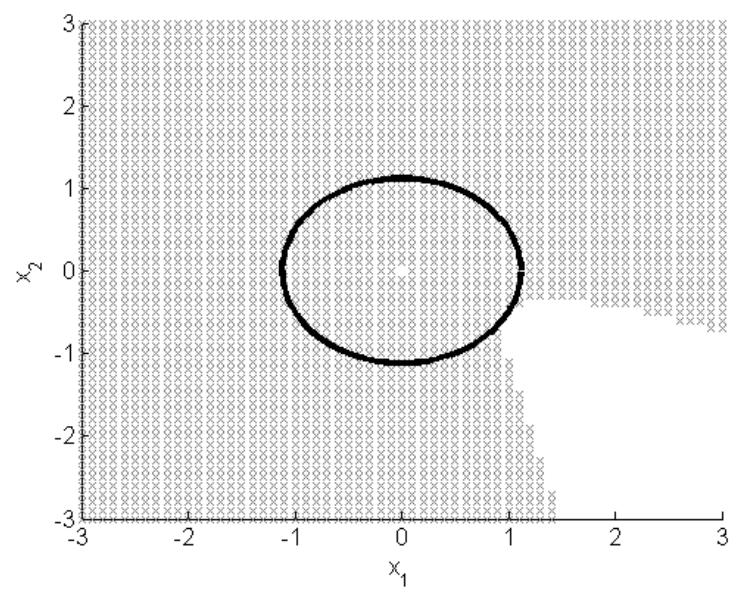

(a) Stability region estimated by Bracci et al. [4]

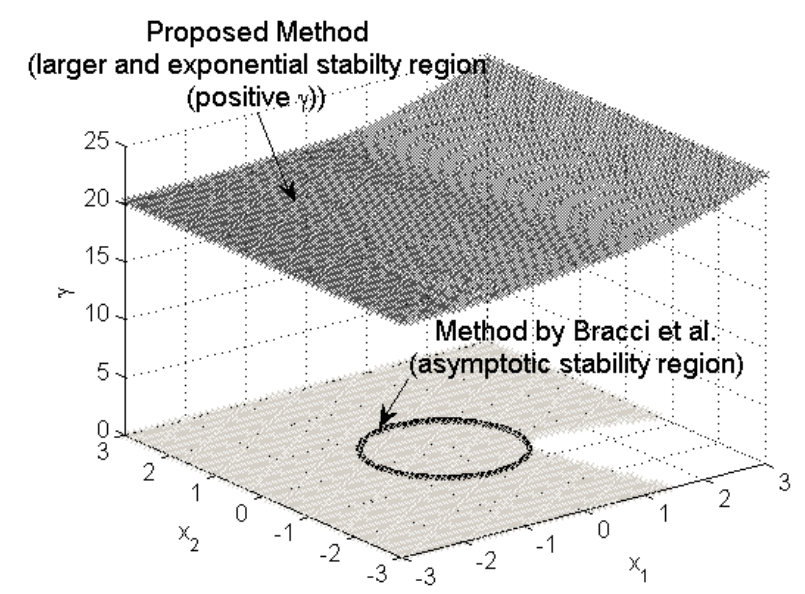

(b) Estimated stability region and convergence rate $(\gamma)$ for the proposed method (the upper surface)

Fig. 3. Comparison of the stability region estimates for Example 2

circle denote the area satisfying the Lyapunov local stability theorem and the estimated stability region for the SDREcontrolled system, respectively. Fig. 3(b) shows the results for the proposed method. Here, the stability region was not added explicitly by using a circle because the convergence rate has positive values in the whole region, which indicates that the system has a bigger and exponentially stable region. The upper surface in the figure shows the variation of the value of the convergence rate $\gamma$ with respect to $x$. It should be noted that the estimated stability region by the proposed method could be more accurate in the sense that the method considers the partial derivative terms in (29) and the state-dependent $M(x)$ in (20), while the aforementioned work ignores the terms and uses a constant $M^{\prime}$ in (28), which is obtained from the equilibrium point. Note that the larger stability region in the this example is just one of the results. The estimated regions could be smaller than the other methods. However the important thing is that the result is more reliable than those from the other methods.

From the two examples, the superiority of the proposed 
method for estimating the exponential stability region for the SDRE feedback systems is apparent. Note that the proposed method uses more accurate information than the prior work, so that the results could be more reliable.

\section{CONCLUSIONS}

We proposed a new method, motivated by contraction analysis, to estimate the stability region for the nonlinear system controlled by the SDRE controllers. The proposed method estimates the exponential stability region for the SDRE feedback systems, while previous relevant work estimated the asymptotically stable regions in a conservative manner. The proposed method considers the variation of $P(x)$ and $M(x)$ with respect to the states, while the prior work neglected the effect, which could lead to instability in the systems. Moreover, this method also shows how rapidly the controlled system is stabilized exponentially in each region with the given states, which is critical information for determining the stability characteristics. Through two examples, we demonstrated the superiority of the proposed method for estimating the stability region for nonlinear SDRE feedback systems.

There might still be a discrepancy between the true stability region and the one estimated by the proposed method because of the effect of $\dot{M}(x)$. We assumed the effect to be negligible by the assumption that $\dot{x}$ is sufficiently small and $\partial M(x) / \partial x$ is bounded. However the value of $\dot{M}(x)$ can be obtained numerically, so that the effect can be measured. Therefore, the assumption could be justified using numerical data. For more rigorous estimates, further work is needed.

\section{ACKNOWLEDGMENTS}

This work was supported by the Air Force Office of Scientific Research (AFOSR). The authors thank Prof. J. Oliver at the Virtual Reality Application Center, Iowa State University for his support. The authors gratefully acknowledge stimulating discussions with Prof. J.-J. E. Slotine, Dr. W. Lohmiller, and A. A. Paranjape.

\section{REFERENCES}

[1] E. M. Aylward, P. A. Parrilo, and J.-J. E. Slotine, "Stability and Robustness Analysis of Nonlinear Systems via Contraction Metrics and SOS Programming," Automatica, Vol. 44, No. 8, 2008, pp. 21632170.

[2] A. Bogdanov and E. Wan, "State-Dependent Riccati Equation Control for Small Autonomous Helicopters," AIAA J. of Guid., Contr., Dyn., Vol. 30, No. 1, 2007, pp. 47-60.

[3] S. Boyd, L. E. Ghaoui, E. Feron, and V. Balakrishnan, Linear Matrix Inequalities in System and Control Theory SIAM, Philadelphia, PA, 1994.

[4] A. Bracci, M. Innocenti, and L. Pollini, "Estimation of the Region of Attraction for State-Dependent Riccati Equation Controllers," AIAA J. of Guid., Contr., Dyn., Vol. 29, No. 6, 2006, pp. 1427-1430.

[5] I. Chang, S.-Y. Park, and K.-H. Choi, "Decentralized Coordinated Attitude Control for Satellite Formation Flying via the State-Dependent Riccati Equation Technique," Int. J. of Non-Linear Mechanics, Vol. 44, No. 8, 2009, pp. 891-904.

[6] I. Chang, S.-Y. Park, and K.-H. Choi, "Nonlinear Attitude Control of a Tether-Connected Multi-Satellite System in Three-Dimensional Free Space," IEEE Trans. Aerospace and Electronic Sys., (in Press).

[7] S.-J. Chung and J.-J. E. Slotine, "Cooperative Robot Control and Concurrent Synchronization of Lagrangian Systems," IEEE Trans. Robot., Vol. 25, No. 3, 2009, pp. 686-700.
[8] T. Çimen, "State-Dependent Riccati Equation (SDRE) Control: A Survery," in Proc. of the 17th IFAC World Congress, Seoul, Korea, Jun. 2008, pp. 3761-3775.

[9] J. R. Cloutier, "State-Dependent Riccati Equation Technique: An Overview," in Proc. of the Amer. Contr. Conf., Albuquerque, NM, Jun. 1997, pp. 932-936.

[10] J. R. Cloutier and P. H. Zipfel, "Hypersonic Guidance via the StateDependent Riccati Equation Control Method," Proc. of the IEEE Conf. Contr. App., Hawaii, May 1999, pp. 96-91.

[11] J. R. Cloutier and D. T. Stansbery, "Nonlinear, Hybrid Bank-ToTurn/Skid-To-Turn Autopilot Design," in Proc. of the AIAA Guid., Nav., Contr. Conf., Montreal, Canada, Aug. 2001, AIAA 2001-4158.

[12] E. B. Erdem, "Analysis and Real-Time Implementation of StateDependent Riccati Equation Controlled Systems," Ph.D. Dissertation, University of Illinois at Urbana-Champaign, Illinois, 2001, pp. 5-35.

[13] E. B. Erdem and A. G. Alleyne, "Globally Stabilizing Second-Order Nonlinear Systems by SDRE Control", Proc. of the Amer. Contr. Conf., San Diego, CA, Jun. 1999, pp.2501-2505.

[14] E. B. Erdem and A. G. Alleyne, "Estimation of Stability Regions of SDRE Controlled Systems Using Vector Norms," in Proc. of the Amer. Contr. Conf., Anchorage, AK, May 2002, pp. 80-85.

[15] D. J. Irvin and D. R. Jacques, "Linear vs. Nonlinear Control Techniques for the Reconfiguration of Satellite Formations," in Proc. of the AIAA Guid., Nav., Contr. Conf., Montreal, Canada, Aug. 2001, AIAA 2001-4089.

[16] H. K. Khalil, Nonlinear Systems, 3rd ed., Prentice Hall, Upper Saddle River, NJ, 2002, pp. 111-194.

[17] W. Langson and A. Alleyne, "A Stability Result with Application to Nonlinear Regulation," ASME J. of Dyn. Sys., Meas., Contr., Vol. 124, 2002, pp. 452-456.

[18] W. Lohmiller and J.-J. E. Slotine, "On Contraction Analysis for Nonlinear Systems," Automatica, Vol. 34, No. 6, 1998, pp. 683-695.

[19] D. McCaffrey and S. P. Banks, "Lagrangian Manifolds and Asymptotically Optimal Stabilizing Feedback Control," Sys. Contr. Lett., Vol. 43, No. 3, 2001, pp. 219-224.

[20] P. K. Menon and V. R. Iragavarapu, "Integrated Design of Agile Missile Guidance and Control Systems," SBIR Report, Dahlgren, Virginia, 1997.

[21] C. P. Mracek and J. R. Cloutier, "Control Designs for the Nonlinear Benchmark Problem via the State-Dependent Riccati Equation Method," Int. J. Robust Nonlinear Contr., Vol. 8, 1998, pp. 401-433.

[22] P. Parrilo, "Structured Semidefinite Programs and Semialgebraic Geometry Methods in Robustness and Optimization", Ph.D. Dissertation, California Institute of Technology, California, 2000.

[23] P. Seiler, "Stability Region Estimates for SDRE Controlled Systems Using Sum of Squares Optimization," in Proc. of the Amer. Contr. Conf., Denver, CO, Jun. 2003, pp. 1867-1872.

[24] J. S. Sharma and J. R. Cloutier, "Existence of SDRE Stabilizing Feedback," IEEE Trans. Autom. Contr., Vol. 48, No. 3, 2003, pp. 513517.

[25] J.-J. E. Slotine and W. Li, Applied Nonlinear Control, Prentice Hall, Englewood Cliffs, NJ, 1991, pp. 40-99.

[26] S. Suzuki, K. Furuta, A. Sugiki, and S. Hatakeyama, "Nonlinear Optimal Internal Forces Control and Application to Swing-Up and Stabilization of Pendulum," ASME J. of Dyn. Sys., Meas., Contr., Vol. 126, 2004, pp. 568-573. 